Please note that this version of the article is outdated (not peer reviewed). An updated version of this manuscript has been accepted for publication in Consciousness and Cognition. The Version of Record is available at: https://doi.org/10.1016/j.concog.2022.103335.

\title{
Spontaneous Mind-Wandering Tendencies Linked to Cognitive Flexibility in Young Adults
}

\author{
Yi-Sheng Wong ${ }^{\mathbf{1}, 2}$, Adrian R. Willoughby ${ }^{3}$, and Liana Machado ${ }^{\mathbf{1} 2}$ \\ ${ }^{1}$ Department of Psychology and Brain Health Research Centre, University of Otago, \\ Dunedin, New Zealand \\ ${ }^{2}$ Brain Research New Zealand, Auckland, New Zealand \\ ${ }^{3}$ Department of Psychology, Monash University Malaysia, Subang Jaya, Malaysia
}

\begin{abstract}
Author Note
Yi-Sheng Wong, ORCID: https://orcid.org/0000-0002-5752-4679

Adrian R. Willoughby, ORCID: https://orcid.org/0000-0002-4214-2635

Liana Machado, ORCID: https://orcid.org/0000-0002-0856-3831

Correspondence concerning this article should be addressed to Yi-Sheng Wong, Department of Psychology, University of Otago, William James Building, 275 Leith Walk, Dunedin 9016, New Zealand. Email: yishengwong.ysw@ gmail.com
\end{abstract}




\begin{abstract}
Despite that previous studies have investigated mind wandering using task-switching paradigms, the association between the tendency to mind wander and cognitive flexibility remains largely unexplored. The present study investigated the relationship between selfreported spontaneous mind-wandering tendencies and task-switching performance in young adults. Seventy-nine university students performed a forced task-switching and a voluntary task-switching paradigm and then completed a battery of questionnaires. The results showed that compared to participants with lower spontaneous mind-wandering tendencies, participants with higher spontaneous mind-wandering tendencies demonstrated better performance (evidenced by smaller switch cost reaction times) in the forced task-switching paradigm despite indicating more mind wandering during task performance. Performance on the voluntary task-switching paradigm, on the other hand, did not differ between the two groups. The findings in the forced task-switching paradigm indicate a link between mind wandering and cognitive flexibility, thus providing initial evidence in favor of a role for switching in mind wandering.
\end{abstract}

Keywords: task-unrelated thoughts; mental set shifting; task switching; exogenous switching; endogenous switching 


\section{Spontaneous Mind-Wandering Tendencies Linked to Cognitive Flexibility in Young Adults}

\section{Introduction}

In the last two decades there has been a growing interest in the psychological study of mind wandering - a common phenomenon in which our attention is directed away from the task at hand toward task-unrelated thoughts (for reviews, see Kvavilashvili \& Rummel, 2020; Smallwood et al., 2018; Smallwood \& Schooler, 2006; 2015). Understanding the nature of mind wandering is important for several reasons. First, mind wandering often impairs task performance, resulting in slower reaction times and lower accuracy (for reviews, see Mooneyham \& Schooler, 2013; Stan \& Christoff, 2018). Second, in contrast to the first reason, mind wandering has been found to have major benefits such as enhancing creative thinking (e.g., Gable et al., 2019; Zedelius \& Schooler, 2015) and facilitating future planning (e.g., Mazzoni, 2019). Third, distinct patterns of mind wandering may signal a number of clinical conditions (for reviews, see Bozhilova et al., 2018; Kvavilashvili et al., 2020). For example, decreases in the frequency of mind wandering have been reported in Alzheimer's disease (e.g., Gyurkovics et al., 2018) and Parkinson's disease (e.g., Walpola et al., 2020), whereas increases in the frequency of mind wandering have been reported in attentiondeficit/hyperactivity disorder (ADHD; e.g., Bozhilova et al., 2021; Mowlem, Skirrow, et al., 2019). Collectively, these considerations have motivated a growing interest in research investigating what factors regulate the occurrence and maintenance of mind wandering.

Despite that a number of hypotheses have been put forward to explain why mind wandering occurs (e.g., McVay \& Kane, 2009, 2010, 2012; Smallwood, 2013; Smallwood \& Schooler, 2006; Thomson et al., 2015), some findings remain difficult to explain by any one single theory. For example, an increasing number of studies have demonstrated that older adults tend to report less mind wandering than healthy young adults (e.g., Jordão et al., 2019; 
Maillet et al., 2020; Moran et al., 2021; for a review, see Maillet \& Schacter, 2016). This is puzzling because the occurrence of mind wandering is thought to reflect a failure of executive control to defend primary-task focus (McVay \& Kane, 2009, 2010, 2012), yet older adults generally have poorer executive control (for reviews, see Craik \& Salthouse, 2011; Machado, 2021; McDonald et al., 2018), therefore one would expect the frequency of mind wandering to increase as people age (due to decreased executive control; Thomson et al., 2015). Furthermore, mind-wandering studies involving task-switching paradigms in healthy young adults have consistently demonstrated that switching performance is not adversely affected by mind wandering (i.e., similar reaction times were obtained for the trials leading up to "ontask" and "off-task" reports; Arnau et al., 2020; Kam \& Handy, 2014; Thomson et al., 2014). These findings are perplexing because (a) mind wandering is conceived as a resourcedemanding process that should result in impaired task performance due to insufficient cognitive resources being devoted to the task at hand (Smallwood, 2010; Smallwood \& Schooler, 2006; Thomson et al., 2015); and (b) mind wandering has repeatedly been found to impair performance on tasks that tap the other core executive control measures-working memory (e.g., Goller et al., 2020; Schurer et al., 2020; Soemer \& Schiefele, 2020) and inhibition (e.g., Banks \& Welhaf, 2021; Groot et al., 2021; Martínez-Pérez et al., 2021). Taken together, these findings suggest existing theories of mind wandering may need to be updated to account for these experimental findings.

To address this, we recently outlined a view of mind wandering that takes into consideration cognitive flexibility, proposing that instances of mind wandering are in fact instances of mental set shifting (Wong et al., 2021; for a similar argument, see Murray \& Krasich, 2020). Cognitive flexibility, or mental set shifting, is one of the core executive functions that enables a person to coordinate thoughts and actions in response to changing demands or priorities (Buttelmann \& Karbach, 2017; Diamond, 2013; Miyake et al., 2000). 
We suggest that mind wandering requires cognitive flexibility (Wong et al., 2021), as it involves a shift from mental sets that are related to the task at hand toward task-unrelated mental sets (Smallwood \& Schooler, 2006). According to this account, there should be an association between mind-wandering tendencies and cognitive flexibility, such that individuals who mind wander more frequently should show better mental set shifting abilities.

In the current study, we investigated this newly formulated switching account of mind wandering in a group of young adults. To assess task-switching abilities and taskswitching tendencies, we used two task-switching paradigms, one involving forced random task switches (forced task switching; Sudevan \& Taylor, 1987) and the other involving selfinitiated voluntary task switches (voluntary task switching; Arrington \& Logan, 2004). Based on previous studies (Seli, Carriere, et al., 2015; Seli et al., 2016), we treated the tendency to mind wander as a personality trait, which was assessed using the Mind Wandering: Deliberate (MW-D) and Spontaneous (MW-S) self-report scales (Carriere et al., 2013). In several studies of mind wandering, these scales, particularly the MW-S, were found to be valid and reliable instruments for measuring trait-level mind wandering (e.g., Mowlem, Agnew-Blais, et al., 2019; Seli, Smallwood, et al., 2015). Additionally, we measured mind wandering during performance of the task-switching paradigms using the Thinking Content Component of the Dundee Stress State Questionnaire (DSSQ; Matthews et al., 2002), which is a self-report questionnaire widely used to assess mind-wandering frequency (e.g., Barron et al., 2011; Helton \& Warm, 2008; Neigel et al., 2019; Smallwood et al., 2009), and a Numerical Rating Scale (NRS) for task-unrelated thoughts, which was purpose-designed for this experiment to measure the frequency of mind wandering during performance of the taskswitching paradigms and to confirm the mind-wandering reports collected via the DSSQ using a single direct question. We also measured participants' attentional control abilities 
using the Attentional Control Scale (ACS; Derryberry \& Reed, 2002) and their motivation to perform the switching paradigms using a NRS for motivation.

The first aim of this study was to investigate the association between cognitive flexibility and mind-wandering tendencies. Based on the cognitive flexibility perspective (Wong et al., 2021), we hypothesized that relative to individuals with lower self-reported spontaneous mind-wandering tendencies, individuals with higher self-reported spontaneous mind-wandering tendencies would: (a) exhibit better performance on the forced taskswitching paradigm (as reflected by smaller switch cost reaction times [RTs], which were calculated for each participant by subtracting mean correct RTs on repeat trials from mean correct RTs on switch trials); and (b) mind wander more frequently during performance of the switching paradigms. The second aim of the study was to explore whether individuals with higher self-reported spontaneous mind-wandering tendencies would initiate more switches during performance of the voluntary task-switching paradigm. Based on the idea that frequent mind wanderers are more inclined to allocate their cognitive resources to different mental sets (Wong et al., 2021), we hypothesized that relative to individuals with lower self-reported spontaneous mind-wandering tendencies, individuals with higher selfreported spontaneous mind-wandering tendencies would be more likely to opt to switch tasks. Furthermore, we assessed attentional control and motivation to perform the switching paradigms in an effort to provide insight into whether these factors contributed to any group differences in mind-wandering reports.

\section{Method}

\subsection{Participants}

Eighty-four undergraduate students (69 females and 15 males) from the University of Otago completed the experiment in association with a psychology course. All participants 
reported being native English speakers and having normal or corrected-to-normal vision without color blindness. Five participants were excluded from the analysis for the following reasons: history of psychiatric disorders $(n=3)$ and excessive errors (> $3 S D s$ above the mean; $n=2$, in both cases excessive errors during both task-switching paradigms). This resulted in a final sample of 79 participants ( 65 females and 14 males; age: mean $=19.59$ years, $S D=1.61$ years, range $=18$ to 27 years). This study was approved by the University of Otago Human Ethics Committee (reference number: D19/297), and all participants provided written informed consent prior to participation.

The number of participants was based on an a priori power analysis performed using G*power 3.1 (Faul et al., 2009). Using a freely available effect size calculator from Psychometrica (Lenhard \& Lenhard, 2016), we inverted the Pearson's correlation coefficient of -.31 reported by Franklin et al. (2016) for the correlation between an individual's mindwandering score and the individual's implicit learning score (which is similar to the calculation of switch cost RTs; Nissen \& Bullemer, 1987) to a Cohen's $d$ of -0.6521 based on the formula specified by Dunlap (1994): Common Language Effect Size $=\sin ^{-1}(r) / \pi+0.5$. The power analysis indicated that the minimum required for two-tailed independent-samples $t$ tests to achieve $80 \%$ power given an alpha of .05 was 76 , thus indicating that our final sample size of 79 was sufficient.

\subsection{Procedure and Materials}

Participants first performed a forced task-switching paradigm and then performed a voluntary task-switching paradigm, after which they filled out a battery of questionnaires in the order described in the following subsections. The experiment lasted about 50 min per participant: 4 min for the forced task-switching practice block, 18 min for the forced taskswitching experimental block, 18 min for the voluntary task-switching experimental block, and 10 min to complete the questionnaires. 


\subsubsection{Task-Switching Paradigms}

The paradigms and stimuli used were based on Kessler et al. (2009) and were programmed in PsychoPy3 (Peirce et al., 2019). The stimuli were geometric shapes that varied in three dimensions: shape (triangle [shape: triangle, line width: 10] or circle [shape: regular polygon, number of vertices: 100, line width: 10]), color (red [line color: 1, 0, 0] or blue [line color: $0,0,1]$ ), and size (large [size: 0.25 ] or small [size: 0.10$])$. The shapes always appeared at the center of the screen (position: 0,0) and were drawn on a grey background. The words "Shape", "Colour", and "Size" served as task cues, indicating the dimension to be responded to. The cues were displayed in white (font style: Arial, letter height: 0.045) and were positioned just above the shapes and centered horizontally (position: $0,0.17$ ).

Participants were tested individually in a dimly lit room, seated in front of a 24-inch monitor at a distance of approximately $57 \mathrm{~cm}$. The paradigms began with verbal instructions along with visual illustrations of the stimuli and response mappings. On each trial, participants were instructed to decide, based on the cue presented above the shape, whether the shape was a triangle or a circle (shape cue), whether it was red or blue (color cue), or whether it was large or small (size cue), by pressing the appropriate key ("Z" or "M") on a QWERTY computer keyboard with their left or right index finger, respectively (see Figure 1 for an example trial). Participants were also instructed (similar to Kessler et al., 2009): "Please be accurate in your responses." 


\section{Figure 1}

Schematic Representation of an Example Trial During the Task-Switching Paradigms.

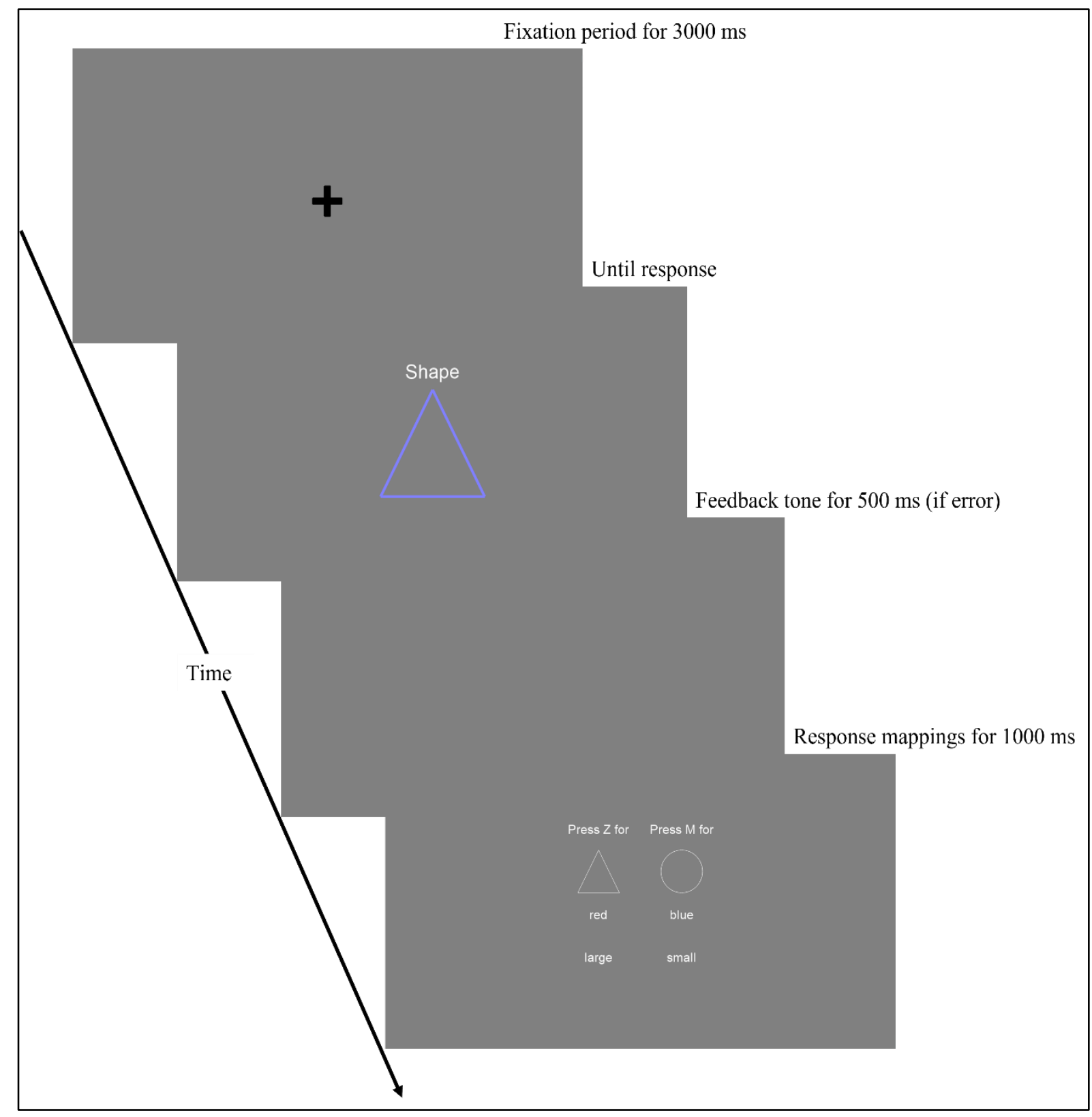

Note. The voluntary task-switching paradigm was identical to the forced task-switching paradigm, except that the task only switched if the participant pressed the spacebar during the fixation period.

Each trial began with the presentation of a black fixation cross (shape: cross, line width: 3, size: 0.07 ) for $3000 \mathrm{~ms}$, followed by the simultaneous presentation of the cue and 
the shape. Whenever participants pressed the wrong button, feedback (pure tone at $350 \mathrm{~Hz}$ for $500 \mathrm{~ms}$ ) was given. The mapping between the stimulus dimensions and the response keys appeared on the screen as the final stimulus event for each trial $(1000 \mathrm{~ms})$. The next trial began after the mapping disappeared. Participants first completed 36 practice trials, followed by 180 experimental trials of the forced task-switching paradigm and then 180 experimental trials of the voluntary task-switching paradigm.

In the forced task-switching paradigm only, the task sequences were pseudorandomized with the constraint that the following were equally probable: (a) shapes (triangle, circle), colors (red, blue), and sizes (large, small); (b) the three possible cue words; and (c) task-switch trials (e.g., from previous trial "Size" task to current trial "Shape" task) and taskrepeat trials (e.g., from previous trial "Size" task to current trial "Size" task). In order to control for sequence difficulty and enable direct comparison of task-switching performance across participants, all participants received the same pseudo-randomized trial order and stimulus-response mappings for both the practice and the experimental blocks.

The voluntary task-switching paradigm was identical to the forced task-switching paradigm, except participants were instructed that, if they wished, they could switch to one of the other two tasks by pressing the space bar during fixation cross presentation. They were informed that by pressing the space bar, one of the other two tasks would be selected randomly. For instance, if the current task was "Colour" and the participant pressed the space bar during the fixation period, the task would be switched to either "Shape" or "Size". Finally, participants were further instructed, similar to Kessler et al. (2009), that "If you wish to respond to the same task, do not press anything when the fixation cross is shown". The first trial was set to the "Size" task. 


\subsubsection{Attentional Control Scale (ACS)}

The ACS (Derryberry \& Reed, 2002) is a 20-item scale used to assess attentional control. Participants responded to statements such as, "I can quickly switch from one task to another" on a 4-point Likert scale, ranging from 1 (almost never) to 4 (always). Participants were instructed to select the response that most accurately reflects their everyday experience. A higher score on the ACS indicates better attentional control. In the current study $(N=79)$, the ACS demonstrated good internal consistency (Cronbach's alpha $=0.76$ ).

\subsubsection{Mind Wandering: Deliberate (MW-D) and Spontaneous (MW-S) Scales}

Deliberate (intentional) and spontaneous (unintentional) mind wandering were assessed with the MW-D and MW-S scales (Carriere et al., 2013). Both scales have four items scored on a 7-point Likert scale, ranging from 1 (rarely/not at all true/almost never) to 7 (a lot/very true/almost always). Participants were instructed to select the answer that most accurately reflects their everyday experience. A higher score on the MW-D scale items (e.g., I allow my thoughts to wander on purpose) indicates a greater tendency to deliberately engage in mind wandering in everyday life, and a higher score on the MW-S scale items (e.g., I mind wander even when I'm supposed to be doing something else) indicates a greater tendency to spontaneously engage in mind wandering in everyday life. In the current study ( $N$ =79), both the MW-D scale items (Cronbach's alpha =0.81) and the MW-S scale items $($ Cronbach's alpha $=0.70)$ demonstrated good internal consistency.

\subsubsection{Numerical Rating Scales (NRSs)}

The NRSs, which were purpose-designed for this experiment, required participants to rate the following questions on an 11-point scale, ranging from 0 (not at all) to 10 (very): “How motivated were you to perform the experiment?", "How frequently were you thinking about things unrelated to the task (e.g., personal reflections)?", and "How frequently were you thinking about things related to the task (e.g., I should respond quicker!)?” The highest 
score (10) indicates highly motivated/more task-unrelated thoughts (TUTs)/more task-related thoughts (TRTs), whereas the lowest score (0) indicates not at all motivated/no TUT/no TRT. The second and third questions were included to confirm the mind-wandering reports collected via the DSSQ.

\subsubsection{Awareness of Self-Initiated Task-Switches}

Participants were asked to indicate "Regarding the voluntary task-switching paradigm, every time I pressed the 'SPACEBAR' to switch tasks, I was consciously aware of it." The response options included "Yes, 100\%", "Yes, but not 100\%", and "Not applicable, as I did not switch task". This question served to explore whether all included participants were consciously aware of the switches they initiated.

\subsubsection{Dundee Stress State Questionnaire (DSSQ)}

Consistent with previous studies (e.g., Barron et al., 2011; Helton \& Warm, 2008; Neigel et al., 2019; Smallwood et al., 2009), the Thinking Content Component of the DSSQ (Matthews et al., 2002) was used to retrospectively assess mind wandering. This scale includes 16 items scored on a 5-point Likert scale, ranging from 1 (never) to 5 (very often). Participants were instructed to indicate how often they had each type of thought while performing the task-switching paradigms. A higher score on TRT subscale items (e.g., I thought about my level of ability) indicates more focus on the task, whereas a higher score on TUT subscale items (e.g., I thought about personal worries) indicates more mind wandering during performance of the switching paradigms. In the current sample $(N=79)$, both the DSSQ-TRT subscale items (Cronbach's alpha $=0.70,8$ items) and the DSSQ-TUT subscale items (Cronbach's alpha $=0.85,8$ items) demonstrated good internal consistency. 


\subsection{Statistical Analyses}

All statistical analyses were performed using SPSS v26 (IBM Corp., Armonk, NY, USA). In order to directly compare participants' task-switching performance, we divided participants into high and low mind-wandering tendency groups on the basis of a median split (similar to Biederman et al., 2017; Franklin et al., 2016; Zhang \& Kumada, 2018) of MW-S scores. This split was based on the MW-S score (rather than the MW-D, the NRS-TUT, or the DSSQ-TUT scores) because it provided the only measure of trait-level spontaneous mind wandering in the current study.

For both switching paradigms, independent $t$ tests analyzed mean correct RTs, switch cost RTs (i.e., mean correct RTs on switch trials minus mean correct RTs on repeat trials), proportion of incorrect responses (i.e., error rates), and switch cost error rates (i.e., mean error rates on switch trials minus mean error rates on repeat trials), with spontaneous mind-wandering tendency group (high, low) as a between-subject variable. The first trial (neither a repeat nor a switch trial) was removed from analyses. For the forced task-switching paradigm only, similar to Kam and Handy (2014), we excluded from further analysis: trials with RTs less than 200 ms or more than three standard deviations above an individual's mean RT. For the voluntary task-switching paradigm only, independent $t$ tests also analyzed number of task switches, and similar to Mittelstädt et al. (2019), we excluded from further analysis: trials with RTs less than 200 ms or greater than $3000 \mathrm{~ms}$. We did not apply the same exclusion criteria for the voluntary task-switching paradigm because all RTs exceeding three standard deviations above the individual's mean RT were self-initiated switch trials. Thus, excluding them would remove meaningful data. Similar to Proskovec et al. (2019), in the current study, participants with smaller switch cost RTs are considered to have better taskswitching performance. 
Independent $t$ tests assessed between-group differences in the MW-S, ACS, MW-D, NRSs, and DSSQ scores. An alpha of .05 was chosen a priori to signify statistical significance. We report Hedges' $g$ as an effect-size estimate for all $t$ tests as it is robust to unequal group sizes (Lakens, 2013). Benchmarks of 0.20, 0.50, 0.80 were used to indicate small, medium, and large effect sizes (Cohen, 1988).

\section{Results}

The mean MW-S scores for the high ( $n=35,83 \%$ females and $17 \%$ males) and low ( $n=44,82 \%$ females and $18 \%$ males) spontaneous mind-wandering tendency groups were $23.20(S D=1.80$, range $=21-28)$ and $16.34(S D=3.40$, range $=7-20)$, respectively, $t(67.923)=11.525, p<.001,95 \% C I[5.671,8.047]$, Hedges' $g=2.419$. No significant difference was observed between the two groups for age $(19.37 \pm 1.59$ years for the high MW-S group and $19.77 \pm 1.63$ years for the low MW-S group), $t(77)=-1.100, p=.275$, $95 \% C I[-1.128,0.325]$, Hedges' $g=-0.246$, or years of education $(14.56 \pm 1.45$ years for the high MW-S group and 14.78 \pm 1.05 years for the low MW-S group), $t(77)=-0.807, p=$ $.422,95 \%$ CI $[-0.787,0.333]$, Hedges' $g=-0.175$.

\subsection{Forced Task-Switching Paradigm}

Group performance in the forced task-switching paradigm is summarized in Table 1. Prior to the analyses, we removed $0.01 \%$ of the correct trials due to the RT being less than $200 \mathrm{~ms}$ and $1.47 \%$ of the correct trials due to the RT exceeding three standard deviations above the individual's mean RT. Compared to the low MW-S group, the high MW-S group had smaller switch cost RTs, $t(77)=-2.125, p=.037,95 \% C I[-65.406,-2.120]$, Hedges' $g$ $=-0.464$, and higher error rates on repeat trials, $t(54.053)=2.175, p=.034,95 \% C I[0.061$, 1.497], Hedges' $g=0.515$. No significant group differences were observed for repeat trial RTs, $t(77)=0.406, p=.686,95 \%$ CI $[-75.160,113.674]$, Hedges' $g=0.090$, switch trial 
RTs, $t(77)=-0.268, p=.789,95 \% C I[-122.849,93.689]$, Hedges' $g=-0.062$, error rate on switch trials, $t(77)=0.600, p=.550,95 \% C I[-1.050,1.955]$, Hedges' $g=0.134$, or switch cost error rates, $t(77)=-0.447, p=.656,95 \% C I[-1.791,1.134]$, Hedges' $g=-0.101$.

\section{Table 1}

Forced Task-Switching Paradigm Performance by Spontaneous Mind-Wandering Tendency Group

\begin{tabular}{|c|c|c|c|c|c|c|c|}
\hline \multirow[b]{2}{*}{ Mind- } & \multicolumn{3}{|c|}{ Mean correct RTs (ms) } & \multirow[b]{2}{*}{ Switch } & \multicolumn{3}{|c|}{ Error rate $(\%)$} \\
\hline & Repeat & Switch & Switch & & Repeat & Switch & Switch \\
\hline wandering & trial & trial & cost & frequency & trial & trial & cost \\
\hline group & $(S D)$ & $(S D)$ & $(S D)$ & $(S D)$ & $(S D)$ & $(S D)$ & $(S D)$ \\
\hline High MW-S & 1072 & 1174 & 102 & 90 & 1.92 & 5.07 & 3.15 \\
\hline$(n=35)$ & $(225)$ & $(250)$ & $(52)$ & (0) & $(1.85)$ & $(2.91)$ & $(2.59)$ \\
\hline Low MW-S & 1053 & 1189 & 135 & 90 & 1.14 & 4.62 & 3.48 \\
\hline$(n=44)$ & (196) & $(232)$ & $(82)$ & (0) & $(1.15)$ & $(3.63)$ & $(3.68)$ \\
\hline Difference & 19 & -15 & $-33 *$ & $\mathbf{0}$ & $0.78 *$ & 0.45 & -0.33 \\
\hline
\end{tabular}

Note. $\mathrm{MW}-\mathrm{S}=$ Mind Wandering-Spontaneous Scale; Repeat trial RTs = mean correct reaction times in repeat trials; Switch trial RTs = mean correct reaction times in switch trials; Switch cost RTs $=$ mean correct reaction times in switch trials - mean correct reaction times in repeat trials; Switch frequency $=$ frequency of task switches; Repeat trial error rate $=$ proportion of incorrect responses in repeat trials; Switch trial error rate $=$ proportion of incorrect responses in switch trials; Switch cost error rate $=$ mean error rate in switch trials mean error rate in repeat trials.

$* p<.05$. 


\subsection{Voluntary Task-Switching Paradigm}

Group performance in the voluntary task-switching paradigm is summarized in Table 2. Prior to the analyses, we removed $0.35 \%$ of the correct trials due to the RT exceeding $3000 \mathrm{~ms}$. There were five participants (two in the high MW-S group and three in the low MW-S group) who did not switch tasks at all (i.e., performed the "Size" task only). Thus, these five participants did not contribute any data to the analysis of switch trial RTs, switch cost RTs, switch trial error rates, and switch cost error rates. No significant difference was observed between the two groups for any of the measures (repeat trial RTs, $t(77)=$ $0.116, p=.908,95 \% C I[-63.762,71.637]$, Hedges' $g=0.026$, switch trial RTs, $t(72)=$ $0.196, p=.845,95 \% C I[-136.589,166.397]$, Hedges' $g=0.046$, switch cost $\mathrm{RTs}, t(72)=$ $0.170, p=.865,95 \% C I[-131.978,156.592]$, Hedges' $g=0.042$, switch frequency, $t(77)=$ $-0.970, p=.335,95 \%$ CI $[-5.654,1.949]$, Hedges' $g=-0.218$, error rate on repeat trials, $t(77)=-0.628, p=.532,95 \% C I[-0.794,0.413]$, Hedges' $g=-0.141$, error rate on switch trials, $t(72)=-0.015, p=.988,95 \% C I[-3.170,3.122]$, Hedges' $g=-0.003$, and switch cost error rates, $t(72)=0.131, p=.896,95 \%$ CI $[-3.031,3.458]$, Hedges' $g=0.030)$. 
Table 2

Voluntary Task-Switching Paradigm Performance by Spontaneous Mind-Wandering Tendency Group

\begin{tabular}{lcccccccc}
\hline & \multicolumn{3}{c}{ Mean correct RTs (ms) } & & \multicolumn{3}{c}{ Error rate (\%) } \\
\cline { 2 - 3 } \cline { 7 - 8 } Mind- & Repeat & Switch & Switch & Switch & Repeat & Switch & Switch \\
wandering & trial & trial & cost & frequency & trial & trial & cost \\
group & $(S D)$ & $(S D)$ & $(S D)$ & $(S D)$ & $(S D)$ & $(S D)$ & $(S D)$ \\
\hline High MW-S & 647 & $1304^{\mathrm{a}}$ & $663^{\mathrm{a}}$ & 6.94 & 1.24 & $2.95^{\mathrm{a}}$ & $1.64^{\mathrm{a}}$ \\
$(n=35)$ & $(124)$ & $(334)$ & $(301)$ & $(8.54)$ & $(1.16)$ & $(7.53)$ & $(7.86)$ \\
Low MW-S & 643 & $1289^{\mathrm{b}}$ & $650^{\mathrm{b}}$ & 8.80 & 1.43 & $2.97^{\mathrm{b}}$ & $1.43^{\mathrm{b}}$ \\
$(n=44)$ & $(168)$ & $(317)$ & $(316)$ & $(8.34)$ & $(1.46)$ & $(6.05)$ & $(6.14)$ \\
Difference & $\mathbf{4}$ & $\mathbf{1 5}$ & $\mathbf{1 3}$ & $\mathbf{- 1 . 8 6}$ & $\mathbf{- 0 . 1 9}$ & $\mathbf{- 0 . 0 2}$ & $\mathbf{0 . 2 1}$ \\
\hline
\end{tabular}

Note. $\mathrm{MW}-\mathrm{S}=$ Mind Wandering-Spontaneous Scale; Repeat trial RTs = mean correct reaction times in repeat trials; Switch trial RTs = mean correct reaction times in switch trials; Switch cost RTs $=$ mean correct reaction times in switch trials - mean correct reaction times in repeat trials; Switch frequency $=$ frequency of task switches; Repeat trial error rate $=$ proportion of incorrect responses in repeat trials; Switch trial error rate $=$ proportion of incorrect responses in switch trials; Switch cost error rate $=$ mean error rate in switch trials mean error rate in repeat trials.

a Data from 33 participants.

${ }^{\mathrm{b}}$ Data from 41 participants.

\subsection{ACS, MW-D, NRSs, Awareness of Self-Initiated Task-Switches, and DSSQ}

Questionnaire scores are summarized by spontaneous mind-wandering tendency group in Table 3. Compared to the low MW-S group, the high MW-S group indicated poorer 
attentional control, $t(77)=-2.511, p=.014,95 \% C I[-6.967,-0.805]$, Hedges' $g=-0.564$, higher deliberate mind-wandering tendencies, $t(77)=2.013, p=.048,95 \% C I[0.023,4.353]$, Hedges' $g=0.452$, higher NRS-TUT, $t(77)=3.560, p=.001,95 \%$ CI $[0.874,3.091]$, Hedges' $g=0.796$, and higher DSSQ-TUT, $t(77)=2.988, p=.004,95 \% C I[1.473,7.355]$, Hedges' $g$ $=0.670$. We did not observe any significant between-group differences in NRS-motivation, $t(77)=-0.259, p=.796,95 \% C I[-0.864,0.665]$, Hedges' $g=-0.058$, NRS-TRT, $t(77)=$ $-1.203, p=.233,95 \% C I[-1.650,0.407]$, Hedges' $g=-0.269$, or DSSQ-TRT, $t(77)=1.632$, $p=.107,95 \%$ CI $[-0.437,4.408]$, Hedges' $g=0.367$. 
Table 3

Questionnaire Scores by Spontaneous Mind-Wandering Tendency Group

\begin{tabular}{|c|c|c|c|c|c|c|c|}
\hline Mind- & ACS & MW-D & NRS- & NRS- & NRS- & DSSQ- & DSSQ- \\
\hline wandering & $(S D)$ & $(S D)$ & Motivation & TRT & TUT & TRT & TUT \\
\hline group & & & $(S D)$ & $(S D)$ & $(S D)$ & $(S D)$ & $(S D)$ \\
\hline High MW-S & 49.11 & 22.14 & 6.51 & 6.63 & 6.91 & 26.49 & 20.80 \\
\hline$(n=35)$ & $(7.24)$ & $(5.13)$ & (1.92) & $(2.40)$ & $(2.36)$ & $(5.56)$ & $(6.52)$ \\
\hline Low MW-S & 53.00 & 19.95 & 6.61 & 7.25 & 4.93 & 24.50 & 16.39 \\
\hline$(n=44)$ & $(6.49)$ & $(4.52)$ & (1.50) & $(2.18)$ & $(2.54)$ & $(5.21)$ & $(6.52)$ \\
\hline Difference & $-3.89 *$ & $2.19 *$ & -0.10 & -0.62 & $1.98 * *$ & 1.99 & $4.41 * *$ \\
\hline \multicolumn{8}{|c|}{ Note. $\mathrm{MW}-\mathrm{S}=$ Mind Wandering-Spontaneous Scale; $\mathrm{ACS}=$ Attentional Control Scale; MW } \\
\hline \multicolumn{8}{|c|}{$\mathrm{D}=$ Mind Wandering-Deliberate Scale; NRS-Motivation = Numerical Rating Scale- } \\
\hline \multicolumn{8}{|c|}{ Motivation; NRS-TRT = Numerical Rating Scale-Task-Related Thought; NRS-TUT = } \\
\hline \multicolumn{8}{|c|}{ Numerical Rating Scale-Task-Unrelated Thought; DSSQ-TRT = Dundee Stress State } \\
\hline \multicolumn{8}{|c|}{ Questionnaire-Task-Related Thought; DSSQ-TUT = Dundee Stress State Questionnaire- } \\
\hline \multicolumn{8}{|c|}{ Task-Unrelated Thought. } \\
\hline$* p<.05$. & & & & & & & \\
\hline
\end{tabular}

Regarding the awareness of self-initiated task-switches, all participants selected "Yes, 100\%" (including those who did not switch tasks at all), except for three participants who selected "Yes, but not 100\%" and pressed the spacebar 25, six, and five times, respectively. As we were unable to identify switches initiated without awareness, no switch trials were removed. 


\section{Discussion}

The purpose of this study was to examine whether healthy young adults with higher self-reported spontaneous mind-wandering tendencies demonstrate better performance on the forced task-switching paradigm and initiate more switches during performance of the voluntary task-switching paradigm. Consistent with our hypothesis, the results showed that compared to participants with lower self-reported spontaneous mind-wandering tendencies, participants with higher self-reported spontaneous mind-wandering tendencies demonstrated smaller switch cost RTs in the forced task-switching paradigm despite indicating more mind wandering during performance of the switching paradigms. The higher levels of mind wandering during the switching paradigms could not be attributed to lower motivation, but could at least in part reflect poorer ability to maintain the current task goal (McVay \& Kane, $2009,2010,2012)$. However, in contradiction to our hypothesis, the number of self-initiated task switches on the voluntary task-switching paradigm did not differ between the two mindwandering tendency groups.

Our findings that participants with higher self-reported spontaneous mind-wandering tendencies demonstrated smaller switch cost RTs in the forced task-switching paradigm despite indicating more mind wandering during the tasks may provide insight into the lack of performance costs associated with mind wandering in previous task-switching studies (e.g., Arnau et al., 2020; Kam \& Handy, 2014; Thomson et al., 2014): mind wandering (i.e., switching between task-related and task-unrelated mental sets) during task-switching performance (i.e., a context in which one is required to switch between task-related mental sets) may in fact promote cognitive flexibility (for a review, see Dreisbach \& Fröber, 2019). Consistent with this conjecture, a recent study provided evidence that when task/mental sets change more frequently, an increase in cognitive flexibility is triggered to improve task-set reconfiguration, which can lead to better task-switching performance (Zhuo et al., 2021). In 
relation to this, we propose that in our study, participants with higher self-reported spontaneous mind-wandering tendencies, who also indicated higher rates of mind wandering during performance of the switching paradigms, may have triggered an increase in cognitive flexibility to a greater extent than participants with lower self-reported spontaneous mindwandering tendencies. Taken together, the current results provide initial empirical evidence suggesting that when those with higher spontaneous mind-wandering tendencies mind wander during performance of a forced task-switching paradigm, they may engage in a "setswitching mode" and consequently can maintain, if not improve, task switching performance.

The failure to support our hypothesis that those with higher self-reported spontaneous mind-wandering tendencies would initiate more switches during performance of the voluntary task-switching paradigm (due to a higher propensity for shifting mental sets) may relate to participants in the current study choosing to switch tasks unusually infrequently. In contrast to a previous study (Kessler et al., 2009) that used the same voluntary task switching paradigm and instructions and observed a mean switch rate of $10 \%$ in Experiment 1 (i.e., on average, a self-initiated switch occurred every 10 trials) and 13\% in Experiment 2 (i.e., on average, a self-initiated switch occurred every 7.5 trials), we observed a mean switch rate of only $4 \%$ across the two mind-wandering tendency groups (i.e., on average, a self-initiated switch occurred every 22.4 trials). The considerably lower number of self-initiated task switches may have limited our ability to explore the association between spontaneous mind-wandering tendencies and voluntary task-switching behavior. To induce higher levels of voluntary task-switching behavior in an unconstrained environment (i.e., where the participant is allowed to perform the same task as often as they prefer), future research should consider the use of the self-organized task-switching paradigm (Mittelstädt et al., 2018), as in several recent studies, this paradigm was found to be powerful enough to 
induce high levels of voluntary task-switching behavior (a mean switch rate of $16 \%$ to $49 \%$; Mittelstädt et al., 2018; 2019; Mittelstädt et al., 2021).

While the current study offers initial evidence of an interrelationship between mind wandering tendencies and switching abilities (Wong et al., 2021), several limitations are noteworthy. First, we did not collect mind-wandering data immediately after participants completed each paradigm, thereby making it impossible to identify whether participants mind wandered more frequently during performance of a relatively effortless task (i.e., the voluntary task-switching paradigm; Seli et al., 2018; Smallwood et al., 2013; Thomson et al., 2013) or during performance of a relatively effortful task (i.e., the forced task-switching paradigm; Xu \& Metcalfe, 2016). Second, it remains unknown whether the current findings, namely that the high MW-S group demonstrated better switching performance despite indicating poorer attentional control and more mind wandering during performance of the switching paradigms, have relevance to other populations (e.g., children, older adults, and clinical populations). Furthermore, like many psychology studies, our participants were primarily female (e.g., Groot et al., 2021; Martínez-Pérez et al., 2021; Mazzoni, 2019) and restricted to young undergraduates in a single Western, educated, industrialized, rich, and democratic cultural context (for a review, see Henrich, 2020). Therefore, future studies should explore the generalizability of the present findings and further validate, or challenge, the switching account of mind wandering.

In addition, we encourage future researchers to examine the role of inhibitory control and working memory in mind wandering, as executive control functions are moderately associated with one another (Miyake et al., 2000) and cannot operate entirely independently (Diamond, 2013). Determining the interrelationships with these other executive functions may help answer two important questions: first, why some populations experience excessive mind-wandering episodes (e.g., individuals with ADHD; Bozhilova et 
al., 2018), while others experience fewer episodes (e.g., individuals with Parkinson's disease; Walpola et al., 2020); and second, why young adults show a negative correlation between mind-wandering rate and working memory capacity (e.g., McVay \& Kane, 2009, 2012; Randall et al., 2014; Robison \& Unsworth, 2018) given that they also show a negative association between working memory capacity and task-switching performance (Miyake et al., 2000; Oberauer et al., 2003; Shipstead et al., 2015; for more details, see Draheim et al., 2016).

In conclusion, the current study represents the first attempt to test the switching perspective of mind wandering. Notwithstanding the limitations in the representativeness of the sample, the results suggest that healthy young adults with higher self-reported spontaneous mind-wandering tendencies outperform those with lower self-reported spontaneous mind-wandering tendencies when required to switch between tasks, providing initial support to the newly forwarded switching perspective (Wong et al., 2021). Although much research is needed to fully understand the role of cognitive flexibility in mind wandering, this first step towards highlighting the relevance of cognitive flexibility in mind wandering may help researchers towards gaining a more nuanced and holistic understanding of past findings (e.g., Arnau et al., 2020; Kam \& Handy, 2014; Thomson et al., 2014). 


\section{Acknowledgement}

This research did not receive any specific grant from funding agencies in the public, commercial, or not-for-profit sectors. Y.-S.W. acknowledges the receipt of a PhD scholarship from the University of Otago.

We thank Prof Jeff Miller for helpful comments on data analyses, and Dr Marijn Kouwenhoven and Wayne Meighan for comments on the earlier drafts.

We have no known conflict of interest to disclose. 


\section{References}

Arnau, S., Löffler, C., Rummel, J., Hagemann, D., Wascher, E., \& Schubert, A. L. (2020). Inter-trial alpha power indicates mind wandering. Psychophysiology, 57(6), e13581. https://doi.org/10.1111/psyp.13581

Arrington, C. M., \& Logan, G. D. (2004). The cost of a voluntary task switch. Psychological Science, 15(9), 610-615. https://doi.org/10.1111/j.0956-7976.2004.00728.x

Banks, J. B., \& Welhaf, M. S. (2021). Individual differences in dimensions of mind wandering: The mediating role of emotional valence and intentionality. Psychological Research, 1-23. https://doi.org/10.1007/s00426-021-01579-2

Barron, E., Riby, L. M., Greer, J., \& Smallwood, J. (2011). Absorbed in thought: The effect of mind wandering on the processing of relevant and irrelevant events. Psychological Science, 22(5), 596-601. https://doi.org/10.1177/0956797611404083

Biederman, J., Fitzgerald, M., Uchida, M., Spencer, T. J., Fried, R., Wicks, J., Saunders, A., \& Faraone, S. V. (2017). Towards operationalising internal distractibility (Mind Wandering) in adults with ADHD. Acta Neuropsychiatrica, 29(6), 330-336.

\section{https://doi.org/10.1017/neu.2016.70}

Bozhilova, N., Michelini, G., Jones, C., Kuntsi, J., Rubia, K., \& Asherson, P. (2021). Context regulation of mind wandering in ADHD. Journal of Attention Disorders, 25(14), 2014-2027. https://doi.org/10.1177/1087054720956714

Bozhilova, N. S., Michelini, G., Kuntsi, J., \& Asherson, P. (2018). Mind wandering perspective on attention-deficit/hyperactivity disorder. Neuroscience and Biobehavioral Reviews, 92, 464-476. https://doi.org/10.1016/j.neubiorev.2018.07.010

Buttelmann, F., \& Karbach, J. (2017). Development and plasticity of cognitive flexibility in early and middle childhood. Frontiers in Psychology, 8, 1040.

https://doi.org/10.3389/fpsyg.2017.01040 
Carriere, J. S. A., Seli, P., \& Smilek, D. (2013). Wandering in both mind and body: Individual differences in mind wandering and inattention predict fidgeting. Canadian Journal of Experimental Psychology, 67(1), 19-31. https://doi.org/10.1037/a0031438

Cohen, J. (1988). Statistical Power Analysis for the Behavioral Sciences. Routledge Academic.

Craik, F. I. M., \& Salthouse, T. A. (2011). The Handbook of Aging and Cognition. Psychology Press.

Derryberry, D., \& Reed, M. A. (2002). Anxiety-related attentional biases and their regulation by attentional control. Journal of Abnormal Psychology, 111(2), 225-236. https://doi.org/10.1037/0021-843X.111.2.225

Diamond, A. (2013). Executive functions. Annual Review of Psychology, 64, 135-168.

\section{https://doi.org/10.1146/annurev-psych-113011-143750}

Draheim, C., Hicks, K. L., \& Engle, R. W. (2016). Combining reaction time and accuracy: The relationship between working memory capacity and task switching as a case example. Perspectives on Psychological Science, 11(1), 133-155.

\section{https://doi.org/10.1177/1745691615596990}

Dreisbach, G., \& Fröber, K. (2019). On how to be flexible (or not): Modulation of the stability-flexibility balance. Current Directions in Psychological Science, 28(1), 3-9. https://doi.org/10.1177/0963721418800030

Dunlap, W. P. (1994). Generalizing the common language effect size indicator to bivariate normal correlations. Psychological Bulletin, 116(3), 509-511. https://doi.org/10.1037/0033-2909.116.3.509

Faul, F., Erdfelder, E., Buchner, A., \& Lang, A.-G. (2009). Statistical power analyses using G*Power 3.1: Tests for correlation and regression analyses. Behavior Research Methods, 41(4), 1149-1160. https://doi.org/10.3758/BRM.41.4.1149 
Franklin, M. S., Smallwood, J., Zedelius, C. M., Broadway, J. M., \& Schooler, J. W. (2016). Unaware yet reliant on attention: Experience sampling reveals that mind-wandering impedes implicit learning. Psychonomic Bulletin \& Review, 23(1), 223-229. https://doi.org/10.3758/s13423-015-0885-5

Gable, S. L., Hopper, E. A., \& Schooler, J. W. (2019). When the muses strike: Creative ideas of physicists and writers routinely occur during mind wandering. Psychological Science, 30(3), 396-404. https://doi.org/10.1177/0956797618820626

Goller, H., Banks, J. B., \& Meier, M. E. (2020). An individual differences investigation of the relations among life event stress, working memory capacity, and mind wandering: A preregistered replication-extension study. Memory \& Cognition, 48(5), 759-771. https://doi.org/10.3758/s13421-020-01014-8

Groot, J. M., Boayue, N. M., Csifcsák, G., Boekel, W., Huster, R., Forstmann, B. U., \& Mittner, M. (2021). Probing the neural signature of mind wandering with simultaneous fMRI-EEG and pupillometry. NeuroImage, 224, 117412. https://doi.org/10.1016/j.neuroimage.2020.117412

Gyurkovics, M., Balota, D. A., \& Jackson, J. D. (2018). Mind-wandering in healthy aging and early stage Alzheimer's disease. Neuropsychology, 32(1), 89-101. https://doi.org/10.1037/neu0000385

Helton, W. S., \& Warm, J. S. (2008). Signal salience and the mindlessness theory of vigilance. Acta Psychologica, 129(1), 18-25. https://doi.org/10.1016/j.actpsy.2008.04.002

Henrich, J. (2020). The WEIRDest People in the World: How the West Became Psychologically Peculiar and Particularly Prosperous. Penguin UK. 
Jordão, M., Ferreira-Santos, F., Pinho, M. S., \& St Jacques, P. L. (2019). Meta-analysis of aging effects in mind wandering: Methodological and sociodemographic factors. Psychology and Aging, 34(4), 531-544. https://doi.org/10.1037/pag0000356

Kam, J. W., \& Handy, T. C. (2014). Differential recruitment of executive resources during mind wandering. Consciousness and Cognition, 26, 51-63. https://doi.org/10.1016/j.concog.2014.03.002

Kessler, Y., Shencar, Y., \& Meiran, N. (2009). Choosing to switch: Spontaneous task switching despite associated behavioral costs. Acta Psychologica, 131(2), 120-128. https://doi.org/10.1016/j.actpsy.2009.03.005

Kvavilashvili, L., Niedźwieńska, A., Gilbert, S. J., \& Markostamou, I. (2020). Deficits in spontaneous cognition as an early marker of Alzheimer's disease. Trends in Cognitive Sciences, 24(4), 285-301. https://doi.org/10.1016/j.tics.2020.01.005

Kvavilashvili, L., \& Rummel, J. (2020). On the nature of everyday prospection: A review and theoretical integration of research on mind-wandering, future thinking, and prospective memory. Review of General Psychology, 24(3), 210-237. https://doi.org/10.1177/1089268020918843

Lakens, D. (2013). Calculating and reporting effect sizes to facilitate cumulative science: A practical primer for t-tests and ANOVAs. Frontiers in Psychology, 4, 863. https://doi.org/10.3389/fpsyg.2013.00863

Lenhard, W., \& Lenhard, A. (2016). Calculation of Effect Sizes. Dettelbach (Germany): Psychometrica. https://www.psychometrica.de/effect_size.html

Machado, L. (2021). Understanding cognition and how it changes with aging, brain disease, and lifestyle choices. Journal of the Royal Society of New Zealand, 51(1), 128-142. https://doi.org/10.1080/03036758.2020.1796102 
Maillet, D., \& Schacter, D. L. (2016). From mind wandering to involuntary retrieval: Agerelated differences in spontaneous cognitive processes. Neuropsychologia, 80, 142156. https://doi.org/10.1016/j.neuropsychologia.2015.11.017

Maillet, D., Yu, L., Hasher, L., \& Grady, C. L. (2020). Age-related differences in the impact of mind-wandering and visual distraction on performance in a go/no-go task. Psychology and Aging, 35(5), 627-638. https://doi.org/10.1037/pag0000409

Martínez-Pérez, V., Baños, D., Andreu, A., Tortajada, M., Palmero, L. B., Campoy, G., \& Fuentes, L. J. (2021). Propensity to intentional and unintentional mind-wandering differs in arousal and executive vigilance tasks. PloS One, 16(10), e0258734. https://doi.org/10.1371/journal.pone.0258734

Matthews, G., Campbell, S. E., Falconer, S., Joyner, L. A., Huggins, J., Gilliland, K., Grier, R., \& Warm, J. S. (2002). Fundamental dimensions of subjective state in performance settings: Task engagement, distress, and worry. Emotion, 2(4), 315-340. https://doi.org/10.1037/1528-3542.2.4.315

Mazzoni, G. (2019). Involuntary memories and involuntary future thinking differently tax cognitive resources. Psychological Research, 83(4), 684-697. https://doi.org/10.1007/s00426-018-1123-3

McDonald, A. P., D'Arcy, R. C. N., \& Song, X. (2018). Functional MRI on executive functioning in aging and dementia: A scoping review of cognitive tasks. Aging Medicine, 1(2), 209-219. https://doi.org/10.1002/agm2.12037

McVay, J. C., \& Kane, M. J. (2009). Conducting the train of thought: Working memory capacity, goal neglect, and mind wandering in an executive-control task. Journal of Experimental Psychology: Learning, Memory, and Cognition, 35(1), 196-204. https://doi.org/10.1037/a0014104 
McVay, J. C., \& Kane, M. J. (2010). Does mind wandering reflect executive function or executive failure? Comment on Smallwood and Schooler (2006) and Watkins (2008). Psychological Bulletin, 136(2), 188-197. https://doi.org/10.1037/a0018298

McVay, J. C., \& Kane, M. J. (2012). Why does working memory capacity predict variation in reading comprehension? On the influence of mind wandering and executive attention. Journal of Experimental Psychology: General, 141(2), 302-320.

\section{https://doi.org/10.1037/a0025250}

Mittelstädt, V., Miller, J., \& Kiesel, A. (2018). Trading off switch costs and stimulus availability benefits: An investigation of voluntary task-switching behavior in a predictable dynamic multitasking environment. Memory \& Cognition, 46(5), 699-715. https://doi.org/10.3758/s13421-018-0802-Z

Mittelstädt, V., Miller, J., \& Kiesel, A. (2019). Linking task selection to task performance: Internal and predictable external processing constraints jointly influence voluntary task switching behavior. Journal of Experimental Psychology: Human Perception and Performance, 45(12), 1529-1548. https://doi.org/10.1037/xhp0000690

Mittelstädt, V., Schaffernak, I., Miller, J., \& Kiesel, A. (2021). Balancing cognitive and environmental constraints when deciding to switch tasks: Exploring self-reported task-selection strategies in self-organised multitasking. Quarterly Journal of Experimental Psychology, 74(4), 598-609.

\section{https://doi.org/10.1177/1747021820970079}

Miyake, A., Friedman, N. P., Emerson, M. J., Witzki, A. H., Howerter, A., \& Wager, T. D. (2000). The unity and diversity of executive functions and their contributions to complex "Frontal Lobe" tasks: A latent variable analysis. Cognitive Psychology, 41(1), 49-100. https://doi.org/10.1006/cogp.1999.0734 
Mooneyham, B. W., \& Schooler, J. W. (2013). The costs and benefits of mind-wandering: A review. Canadian Journal of Experimental Psychology, 67(1), 11-18. https://doi.org/10.1037/a0031569

Moran, C. N., McGovern, D. P., Warren, G., Grálaigh, R., Kenney, J. P. M., Smeaton, A., \& Dockree, P. M. (2021). Young and restless, old and focused: Age-differences in mindwandering frequency and phenomenology. Psychology and Aging, 36(2), 252-267. https://doi.org/10.1037/pag0000526

Mowlem, F. D., Agnew-Blais, J., Pingault, J.-B., \& Asherson, P. (2019). Evaluating a scale of excessive mind wandering among males and females with and without attentiondeficit/hyperactivity disorder from a population sample. Scientific Reports, 9(1), 3071. https://doi.org/10.1038/s41598-019-39227-w

Mowlem, F. D., Skirrow, C., Reid, P., Maltezos, S., Nijjar, S. K., Merwood, A., Barker, E., Cooper, R., Kuntsi, J., \& Asherson, P. (2019). Validation of Mind Excessively Wandering Scale and the relationship of mind wandering to impairment in adult ADHD. Journal of Attention Disorders, 23(6), 624-634.

\section{https://doi.org/10.1177/1087054716651927}

Murray, S., \& Krasich, K. (2020). Can the mind wander intentionally? Mind \& Language, 1-

\section{2. https://doi.org/10.1111/mila.12332}

Neigel, A. R., Claypoole, V. L., Fraulini, N. W., Waldfogle, G. E., \& Szalma, J. L. (2019). Where is my mind? Examining mind-wandering and vigilance performance. Experimental Brain Research, 237(2), 557-571. https://doi.org/10.1007/s00221-018-

\section{$\underline{5438-5}$}

Nissen, M. J., \& Bullemer, P. (1987). Attentional requirements of learning: Evidence from performance measures. Cognitive Psychology, 19(1), 1-32.

https://doi.org/10.1016/0010-0285(87)90002-8 
Oberauer, K., Suss, H. M., Wilhelm, O., \& Wittmann, W. W. (2003). The multiple faces of working memory: Storage, processing, supervision, and coordination. Intelligence, 31(2), 167-193. https://doi.org/10.1016/S0160-2896(02)00115-0

Peirce, J., Gray, J. R., Simpson, S., MacAskill, M., Hochenberger, R., Sogo, H., Kastman, E., \& Lindelov, J. K. (2019). PsychoPy2: Experiments in behavior made easy. Behavior Research Methods, 51(1), 195-203. https://doi.org/10.3758/s13428-018-01193-y

Proskovec, A. L., Wiesman, A. I., \& Wilson, T. W. (2019). The strength of alpha and gamma oscillations predicts behavioral switch costs. NeuroImage, 188, 274-281. https://doi.org/10.1016/j.neuroimage.2018.12.016

Randall, J. G., Oswald, F. L., \& Beier, M. E. (2014). Mind-wandering, cognition, and performance: A theory-driven meta-analysis of attention regulation. Psychological Bulletin, 140(6), 1411-1431. https://doi.org/10.1037/a0037428

Robison, M. K., \& Unsworth, N. (2018). Cognitive and contextual correlates of spontaneous and deliberate mind-wandering. Journal of Experimental Psychology: Learning, Memory, and Cognition, 44(1), 85-98. https://doi.org/10.1037/xlm0000444

Schurer, T., Opitz, B., \& Schubert, T. (2020). Working memory capacity but not prior knowledge impact on readers' attention and text comprehension. Frontiers in Education, 5, 26. https://doi.org/10.3389/feduc.2020.00026

Seli, P., Carriere, J. S. A., \& Smilek, D. (2015). Not all mind wandering is created equal: Dissociating deliberate from spontaneous mind wandering. Psychological Research, 79(5), 750-758. https://doi.org/10.1007/s00426-014-0617-x

Seli, P., Konishi, M., Risko, E. F., \& Smilek, D. (2018). The role of task difficulty in theoretical accounts of mind wandering. Consciousness and Cognition, 65, 255-262. https://doi.org/10.1016/j.concog.2018.08.005 
Seli, P., Risko, E. F., \& Smilek, D. (2016). Assessing the associations among trait and state levels of deliberate and spontaneous mind wandering. Consciousness and Cognition, 41, 50-56. https://doi.org/10.1016/j.concog.2016.02.002

Seli, P., Smallwood, J., Cheyne, J. A., \& Smilek, D. (2015). On the relation of mind wandering and ADHD symptomatology. Psychonomic Bulletin \& Review, 22(3), 629636. https://doi.org/10.3758/s13423-014-0793-0

Shipstead, Z., Harrison, T. L., \& Engle, R. W. (2015). Working memory capacity and the scope and control of attention. Attention, Perception, \& Psychophysics, 77(6), 1863 1880. https://doi.org/10.3758/s13414-015-0899-0

Smallwood, J. (2010). Why the global availability of mind wandering necessitates resource competition: Reply to McVay and Kane (2010). Psychological Bulletin, 136(2), 202207. https://doi.org/10.1037/a0018673

Smallwood, J. (2013). Distinguishing how from why the mind wanders: A processoccurrence framework for self-generated mental activity. Psychological Bulletin, 139(3), 519-535. https://doi.org/10.1037/a0030010

Smallwood, J., Fitzgerald, A., Miles, L. K., \& Phillips, L. H. (2009). Shifting moods, wandering minds: Negative moods lead the mind to wander. Emotion, 9(2), 271-276. https://doi.org/10.1037/a0014855

Smallwood, J., Gorgolewski, K. J., Golchert, J., Ruby, F. J., Engen, H., Baird, B., Vinski, M. T., Schooler, J. W., \& Margulies, D. S. (2013). The default modes of reading: Modulation of posterior cingulate and medial prefrontal cortex connectivity associated with comprehension and task focus while reading. Frontiers in Human Neuroscience, 7, 734. https://doi.org/10.3389/fnhum.2013.00734

Smallwood, J., Margulies, D., Bernhardt, B., \& Jefferies, E. (2018). Investigating the elements of thought: Toward a component process account of spontaneous cognition. 
In K. Christoff \& K. C. R. Fox (Eds.), The Oxford Handbook of Spontaneous

Thought: Mind-Wandering, Creativity, and Dreaming (pp. 71-83).

https://doi.org/10.1093/oxfordhb/9780190464745.013.34

Smallwood, J., \& Schooler, J. W. (2006). The restless mind. Psychological Bulletin, 132(6), 946-958. https://doi.org/10.1037/0033-2909.132.6.946

Smallwood, J., \& Schooler, J. W. (2015). The science of mind wandering: Empirically navigating the stream of consciousness. Annual Review of Psychology, 66, 487-518. https://doi.org/10.1146/annurev-psych-010814-015331

Soemer, A., \& Schiefele, U. (2020). Working memory capacity and (in)voluntary mind wandering. Psychonomic Bulletin \& Review, 27(4), 758-767.

\section{https://doi.org/10.3758/s13423-020-01737-4}

Stan, D., \& Christoff, K. (2018). Potential clinical benefits and risks of spontaneous thought: Unconstrained attention as a way into and a way out of psychological disharmony. In K. Christoff \& K. C. R. Fox (Eds.), The Oxford Handbook of Spontaneous Thought: Mind-Wandering, Creativity, and Dreaming (pp. 479-491).

\section{https://doi.org/10.1093/oxfordhb/9780190464745.013.45}

Sudevan, P., \& Taylor, D. A. (1987). The cuing and priming of cognitive operations. Journal of Experimental Psychology: Human Perception and Performance, 13(1), 89-103. https://doi.org/10.1037//0096-1523.13.1.89

Thomson, D. R., Besner, D., \& Smilek, D. (2013). In pursuit of off-task thought: Mind wandering-performance trade-offs while reading aloud and color naming. Frontiers in Psychology, 4, 360. https://doi.org/10.3389/fpsyg.2013.00360

Thomson, D. R., Besner, D., \& Smilek, D. (2015). A resource-control account of sustained attention: Evidence from mind-wandering and vigilance paradigms. Perspectives on Psychological Science, 10(1), 82-96. https://doi.org/10.1177/1745691614556681 
Thomson, D. R., Seli, P., Besner, D., \& Smilek, D. (2014). On the link between mind wandering and task performance over time. Consciousness and Cognition, 27, 14-26. https://doi.org/10.1016/j.concog.2014.04.001

Walpola, I. C., Muller, A. J., Hall, J. M., Andrews-Hanna, J. R., Irish, M., Lewis, S. J. G., Shine, J. M., \& O'Callaghan, C. (2020). Mind-wandering in Parkinson's disease hallucinations reflects primary visual and default network coupling. Cortex, 125, 233245. https://doi.org/10.1016/j.cortex.2019.12.023

Wong, Y.-S., Willoughby, A. R., \& Machado, L. (2021). Reconceptualizing mind wandering from a switching perspective (Preprint). PsyArXiv.

https://doi.org/10.31234/osf.io/dnuys

Xu, J., \& Metcalfe, J. (2016). Studying in the region of proximal learning reduces mind wandering. Memory \& Cognition, 44(5), 681-695. https://doi.org/10.3758/s13421$\underline{016-0589-8}$

Zedelius, C. M., \& Schooler, J. W. (2015). Mind wandering “Ahas” versus mindful reasoning: Alternative routes to creative solutions. Frontiers in Psychology, 6, 834. https://doi.org/10.3389/fpsyg.2015.00834

Zhang, Y., \& Kumada, T. (2018). Automatic detection of mind wandering in a simulated driving task with behavioral measures. PloS One, 13(11), e0207092. https://doi.org/10.1371/journal.pone.0207092

Zhuo, B., Zhu, M., Cao, B., \& Li, F. (2021). More change in task repetition, less cost in task switching: Behavioral and event-related potential evidence. European Journal of Neuroscience, 53(8), 2553-2566. https://doi.org/10.1111/ejn.15113 